\title{
Improving Quality of Services of Ad Hoc Network by Working over Link Budgeting and Delay Elimination
}

\author{
Tanu Preet Singh \\ Department of Computer \\ Science \& Engineering, \\ Amritsar College of \\ Engineering \& \\ Technology, \\ Punjab Technical \\ University, Jalandhar, \\ India.
}

\author{
R.K. Singh \\ PhD, Department of \\ Electronics \& \\ Communication \\ University, Uttrakhand \\ Technical University, \\ Dehradun, India.
}

\author{
Vishal Sharma \\ Department of Computer \\ Science \& Engineering, \\ Amritsar College of \\ Engineering \& \\ Technology, \\ Punjab Technical \\ University, Jalandhar, \\ India.
}

\author{
Manmeet Kaur \\ Department of Computer \\ Science \& Engineering, \\ Amritsar College of \\ Engineering \& \\ Technology, \\ Punjab Technical \\ University, Jalandhar, \\ India.
}

\begin{abstract}
MANETs are networks capable of communicating in a set of small, low cost, low power sensing devices. A wireless sensor networks is totally based on the limiting factor i.e. energy consumption. A wireless sensor network consists of large number of sensor nodes distributed or scattered in particular network region. MANETs consist of node that is highly mobile, so in particular the range of the nodes is very important. Each device in a MANETs is free to move independently in any direction, and will therefore change its links to other devices frequently. The energy and the bandwidth of such path are of major concern. The lifetime of the network depends upon these parameters. There has been a paper already published by us termed DSPO that resolved the performance issues regarding the bandwidth efficiency and the energy efficiency. Also it suggested the alternatives to remove the dead state of the mobile nodes. But one of the concerns that arise as a future work of that protocol was handling of delays that may arise and make the network incompetent in terms of the real time performance. In this paper, we are working on the concept of link budget for MANETs. The formula and techniques given in this paper can be used to solve the problem regarding the transmission. Also, in this paper, we explained the simulator developed by us for link budgeting and practical reading of simulations.
\end{abstract}

\section{Keywords}

Delay, link speed, link budget, number of retransmissions, receiver processing energy, SNCP, selective frequency fading, transmitter energy.

\section{INTRODUCTION}

A wireless ad hoc network is based on the nodes that are mobile and have capabilities of communicating each other with packet radios over a shared wireless medium. The limited radio propagation causes the route to be multi hop [1] [2] [7] [8]. The applications of such networks can be search and rescue, automated battlefields, disaster recovery, crowd control and sensor networks. The routing protocol must have the ability to manage the frequent topology changes caused by the mobility of nodes and these need to be efficient as compared on basis of efficiency in terms of bandwidth and power as well as on basis of load transmission [10] [11].
With the advent of On-demand routing, the tables are not maintained and the topological views are also rescued and the routing totally becomes dynamic [11]. Existing on demand routing protocols such as DSR (Dynamic Source Routing), AODV (Ad-hoc on demand distance vector routing) are the shortest path based routing protocols, also these don't consider the packet size and the antenna range of the nodes as a performance metric due to which there is a problem of long delays and congestions in the routing path and the whole set up of the nodal structure enters in to the dead state [10]. Also, on demand protocols that use the shortest paths as performance metric suffer from performance degradation as the network traffic increases [10]. In the paper [6], the energy of the nodes is the major area of concerned for the research to be carried on in this field. The one of the method suggested was CPACL protocol. It stands for cost based power aware routing protocol. In this paper the energy factor of the nodes is taken to be major concern. In this paper, a routing algorithm has been suggested that selects the path form the source to the destination on basis of the path that consumes the least energy [10]. The path selected for this transmission is the best selected path for the particular types of nodes. This means that if a path is defined from node 1 to node 2 by CPACL algorithm, it is the best suited path in all conditions [7] [10]. This protocol is the reactive routing protocol. It maintains the established routes as long as they are needed by the sources. AODV- CPACL uses sequence numbers to ensure the freshness of routes. The route discovery process is initiated whenever; a traffic source needs a route to a destination. Route discovery typically involves a network wide flood of route request packets targeting the destination and waiting for a route reply. It has also been shown that the per node throughput capacity of ad hoc networks with nodes $n$ decreases with $n$ as $\Theta(1 / n \log n)^{1 / 2}$ [3]. The issue regarding this has been shown as the general capacity cost function of channel capacity for arbitrary input alphabets was studied on single link [4]. In the related work the bits per joule capacity of the network is assumed [3]. The tradeoff between energy and the bandwidth has been analyzed under various assumptions on the channel condition and the interference under a linear equidistant relaying network model without considering the energy consumption at the receiver end [5][6][7]. Also the receiver consumption can be improved by using the cross layer design including the effects of the power amplifier used at the transmitter end [8]. The transport efficiency of an ad hoc network was defined considering the transmitter energy and the receiver's processing energy [9] 
[10]. Thus the energy consumption for the packet transmission and the large number of hops is considered [6]. For the networks that have energy as their limiting resource, the network lifetime related to the energy is one of the significant performance metrics [6]. To solve the dead state problem, we have earlier got our paper published that resolved this issue of nodes getting into the dead state. This protocol was termed as DSPO. But there was a concern about delays that may arise due to selection of alternative path that may occur as hindrance for the real time networks that have performance issues with them.

In this paper, we proposed the concept of link budget for MANETs. The formula and techniques given in this paper can be used to solve the problem regarding the transmission. Also, in this paper, we explained the software developed by us for link budgeting and performing live simulations similar to that performed using NS-2 simulator.

\section{SYSTEM MODEL}

The network model we considered comprises of $\mathrm{k}$ number of hops, hops here are the nodes, and the nodes here considered are to be single channel node. This means for $\mathrm{k}$ number of nodes there is $\mathrm{k}$ number of channels. Thus, if two nodes are communicating at a time, then we have k-1 number of relaying nodes in the network model. The distance between the source and the destination is denoted by $\mathrm{d}$. the distance between the relaying nodes can be decided on basis of the dynamic routing considered or it can be given on mathematical computations, this means that the distance between the relaying nodes will be less than the actual distance between the source and destination. Thus, if we consider a constant, let this constant be an then, from the theoretical analysis [6], we obtain that this value is multiplied with the total distance to obtain the actual distance between the relaying nodes then this value should be positive and less than one. Thus, the distance between the relaying nodes will be:

$$
\mathrm{de}=\sum_{n=0}^{k} \alpha \mathrm{d}
$$

The mobility introduces another simple concept. If the mobility of the structure nodes is more, the attenuation has greater effect but if the nodes are considered to be at rest then, the attenuation comes out to be so small that it can be neglected. Thus, the modified formula for the attenuation loss in a network model will be:

$$
\operatorname{Pr}=\beta \frac{\text { Pout }}{d \eta} \zeta \S
$$

Here, is the attenuation loss in the MANETSs, $\beta$ is the antenna constant, $d$ is the end to end distance between source and destination, $\varepsilon$ is the path loss constant such that $2<\varepsilon<4$ and $\delta$ is the mobility factor. The mobility can be computed by analyzing the movement in terms of number of bits transferred per second per meter of the network model. Here, Pout $=$ fo $($ Pin $)$, which is based on the working power amplifier present in each of the node.

\section{DELAY ELIMINATION PROTOCOL}

Delay Elimination Protocol is the delay optimization protocol that implements the SNCP- Sub network control protection for mobile nodes. This protocol maintains the list of all the nodes that communicate or come across the range of a particular transmitting node and then on basis of shortest path and least energy, it selects the most suitable node for relaying purpose. The ring formation is the similar to that which is been applied in the cellular network but the major difference is that the nodes in this case are mobile and it requires the dynamic node management system that could manage and select the dynamic path such that there are zero delays during switching process. The following views of the NAM animator express the overall working of the protocol and thus explain the technique taken at different movements of the node. The Fig 1 to Fig. 5 is the analysis made by running the scripts coded for delay management in NS-2 simulator. The results obtained clearly shows the formation of clusters and the delay factor that can be clearly noticed in the below animator snap shots. The further analysis can be carried out by the study of the animators and the analysis of trace files to form the graphs that could clearly suggest the actual values for delay optimization.

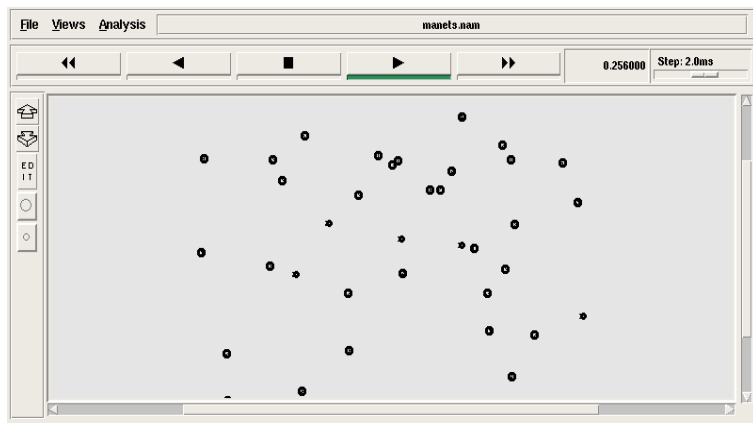

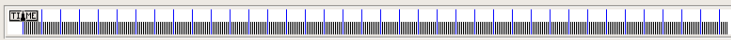

Fig. 1

Fig. 1 is the initial phase of the nodal structure and the next figures explains the movement of nodes and the transmission as wireless medium along with mobility according to the scenario and the traffic generator file scripted for the overall performance of MANETs structure.

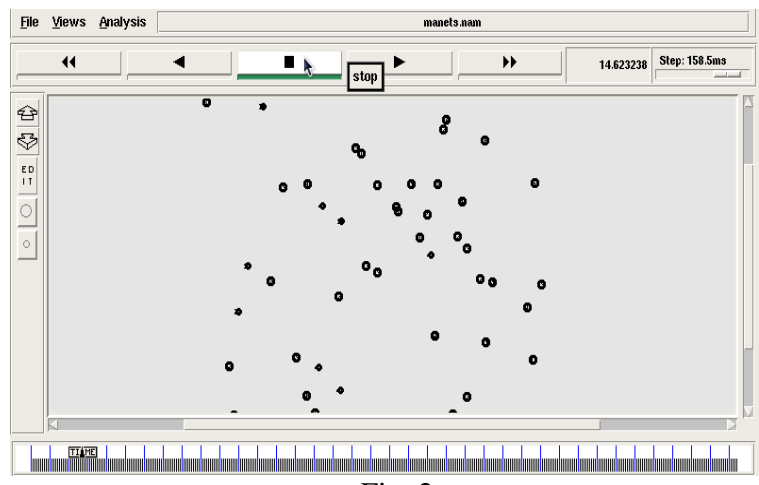

Fig. 2

Fig. 2 shows the movement of the node as compared to the Fig.1 and shows the node moved towards their new destination. 


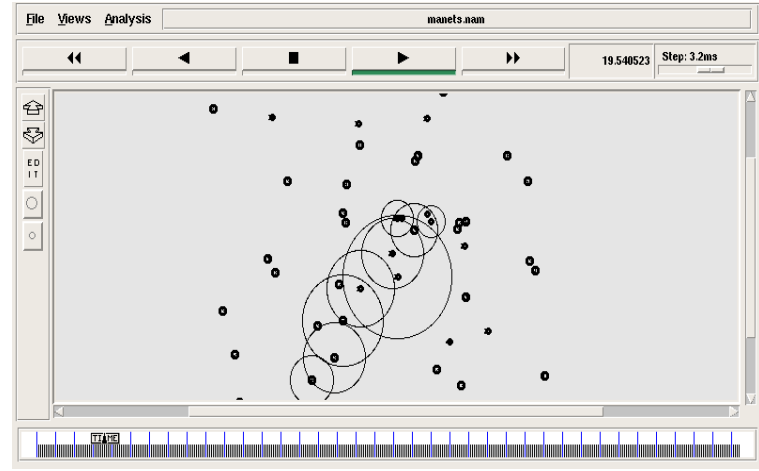

Fig. 3

Fig. 3 shows the transmission process in the mobile node. It should be noted that not all the nodes are transmitting but the number of active connections allowed during the setting up of the traffic generator file can be seen. Also, the nodes causes delay due to cluster formation.

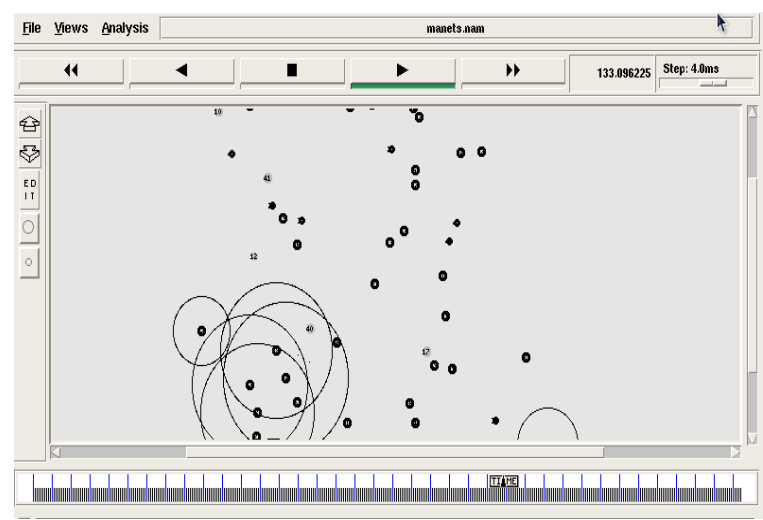

Fig. 4

Fig. 4 shows the weakening of the transmission process along with the movement of nodes. Thus, here is the main requirement of SNCP technique. The SNCP is applied on any one of the node that accounts for constant mobility and the information is stored on each of the relaying node.

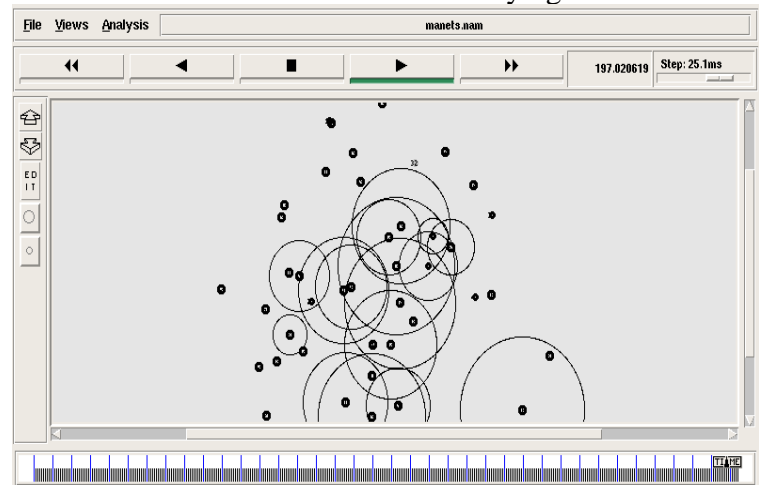

Fig. 5

Fig. 4 shows the final transmission of the nodes along with the mobility effect and the extension of link speed for the purpose of transmission. This accounts for zero delay due to application of SNCP technique. Thus, from the simulations the fault tolerance of the nodal structure is also enhanced.

\subsection{Algorithm for Delay Elimination}

\author{
While (1) \\ \{ \\ Traverse node (i) \\ if ( node $==$ true) \\ \{ \\ then table_entry=valid \\ delay (node) \\ \} else \\ \{ \\ Actual_path=true \\ Exit (0) \\ \} \\ \} \\ Delay (n) \\ \{ \\ For $n=0$ to node \\ \{ \\ If (node_found==true) \\ \{ \\ Apply SNCP, head node \\ Select relay node \\ Maintain record \\ Exit (0) \\ \} \\ Else \\ \{ \\ No change \\ Exit (0) \\ \} \\ \}
}

The above algorithm first traverses all the nodes present in the network structure. After the traversal of nodes, it is verified if most optimum path is selected or not. Then the delay tolerance is checked and on account of delay, the node is selected to act as host for SNCP and the relaying nodes are selected on basis of path that has its energy greater than the threshold energy, i.e. the energy actually required for purpose of transmission.

\subsection{Formula for complexity and asymptotic notation for Delay elimination Algorithm}

In this section, the asymptotic notation of the delay elimination algorithm is studied for bandwidth and energy under transmission at common rate during the entire transmission. Also, the analysis is carried out at common power process of transmission. The results and the mathematical notation for this behavior are shown below.

CASE 1 for non-equidistant hops when $\gamma c=0$ where $\gamma c$ is signal to noise ratio:

$\ln 2$ for $\mathrm{i}=1$ to $\mathrm{k},(\mathrm{a}[\mathrm{i}])^{\wedge} \eta$.

CASE 2 for equidistant hops:

$\left(\mathrm{K}^{\wedge} 1-\eta\right) \ln 2$

Where $\mathrm{k}$ is the number of hops and $\eta$ is pathloss exponent. The above results come out to be infinity when signal to noise ratio is not considered. Also, the results vary for common power due to difference in bandwidth and this difference can 
be seen by calculation of transport efficiency which is the product of efficiencies at common power and common rate.

\subsection{Performance Metrics}

We simulated the typical MANETs for hop and calculated the results for bandwidth, energy, throughput of the network structure taking mobility and the antenna range as the basis of the technique. We have taken values as constant to compute our results. The simulation has been performed for the area ranging over $1500 \times 1500$. Also, the values for the transmitter energy are taken to be $0.38 \mu \mathrm{J}$ and that of receiver processing energy is $0.25 \mu \mathrm{J}$. We concentrated on the following performance metrics:

- Delays: the delay is the latency caused in the process of transmission between the source and the destination. The delay considered in this paper is those that arise due to switching of the path to alternative route.

- Energy Efficiency: Energy efficiency is the efficiency computed over the total energy consumption of the network including the useful energy and the wasted in form of losses during the transmission process. This is calculated as the modified energy for the network structure.

- Bandwidth efficiency: Bandwidth efficiency is the effective rate of transmission that can be considered at the common rate as well as at the common power. The effective rate i.e. the bandwidth is computed over the minimum achievable rate and the number of channel used for the purpose of transmission between the source and the destination. The value considered for minimum achievable rate over which bandwidth efficiency is calculated is 0.25 .

- Throughput: throughput is the number of bits transferred during the transmission process. It is calculated I bits transferred per unit time. For our analysis the time considered is the simulation time.

- Mobility: Mobility is the new parameter that is highlighted in this paper. The readings have been taken by considering the scenario to be mobile and animations has also been recorded for the mobile nodes.

\section{LINK BUDGETING AND PRACTICAL ANALYSIS}

Link budget is method of calculating the overall working of the MANETs structure. This is done through the practical formulas that can be used for calculation of link values that includes values for receiver and the transmitter. This is attained by use of software developed by us that gives the scenario for the evaluation of various link parameters. The general concept regarding the link budgeting is the outage due to frequency selective fading. In case of frequency selective fading, waves from different paths interface with one another at the receiving end. The difference between this propagation of waves can often result in ground reflections of signals, reflections in the ducting layer or propagation in layers having highly positive refractions gradients. More often, the various constituents unite with one another so that a field-strength minimum arises. In this case, there is minimum shift across the frequency band due to atmospheric reflections. The major cause for this shading is in band distortion which can be explained with aid of the transfer function's slope within the frequency band. This whole work can be carried out the software developed by us on the basis of generalized and practical values that can very well judge the working of the current scenario of MANETs. Also, this can be applied to network structure before actually deploying any node for purpose of mobile transmission to check the actual permissible range of the node and the extensions to which it can work. The snap shots of the software are also shown below. Also, the software is being verified by applying it to the scenario of the NS-2 to generate the results that are described in the next section of the paper.

\begin{tabular}{|c|c|c|c|}
\hline \multirow{3}{*}{$\begin{array}{l}\text { Transmitter } \\
\text { Frequency }\end{array}$} & \multicolumn{2}{|l|}{ [rownlink } & \multirow[b]{3}{*}{$\mathrm{MHz}$} \\
\hline & \multicolumn{2}{|l|}{ AN100 } & \\
\hline & \multicolumn{2}{|l|}{3503} & \\
\hline ODU TxPower & 23 & 16 & $\mathrm{dBm}$ \\
\hline T: Antenna Gain & 17 & 24 & $d B i$ \\
\hline Tx Implenentation Loss & 0.5 & 0.5 & $d B$ \\
\hline EIRP : & 39.5 & 39.5 & $\mathrm{dBm}$ \\
\hline \multicolumn{4}{|l|}{ Propagation } \\
\hline Range & \multicolumn{2}{|l|}{10.00} & miles \\
\hline Path Loss & \multicolumn{2}{|l|}{127.41} & $d B$ \\
\hline \multicolumn{4}{|l|}{ Link Profile } \\
\hline Modulation / Coding Rate & 64QAM $3 / 4-$ & 64QAM 2/3 - & \\
\hline Minimum Required CINR & 25 & 23 & $d B$ \\
\hline Expected Channel Throughput & $21 . E$ & 19.2 & $\mathrm{Mb} / \mathrm{s}$ \\
\hline Max Unidirectional Throughput & $18 . ᄃ$ & 16.0 & $\mathrm{Mb} / \mathrm{s}$ \\
\hline \multicolumn{4}{|l|}{ Receiver } \\
\hline Receive Antenna Gain & 24 & 17 & $d B i$ \\
\hline $\mathrm{R} \times$ Implementation Loss & 0.5 & 0.5 & $d B$ \\
\hline RSSI & -64.41 & -71.41 & $\mathrm{dBm}$ \\
\hline Threshold at BER $=10^{-6}$ & -75.0 & -79.0 & $\mathrm{dBm}$ \\
\hline
\end{tabular}

The Fig. 6 shows the software for calculating links performance for the purpose of efficient transmission. The software is also able to calculate the selective frequency fading that can improve the transmission to great extent.

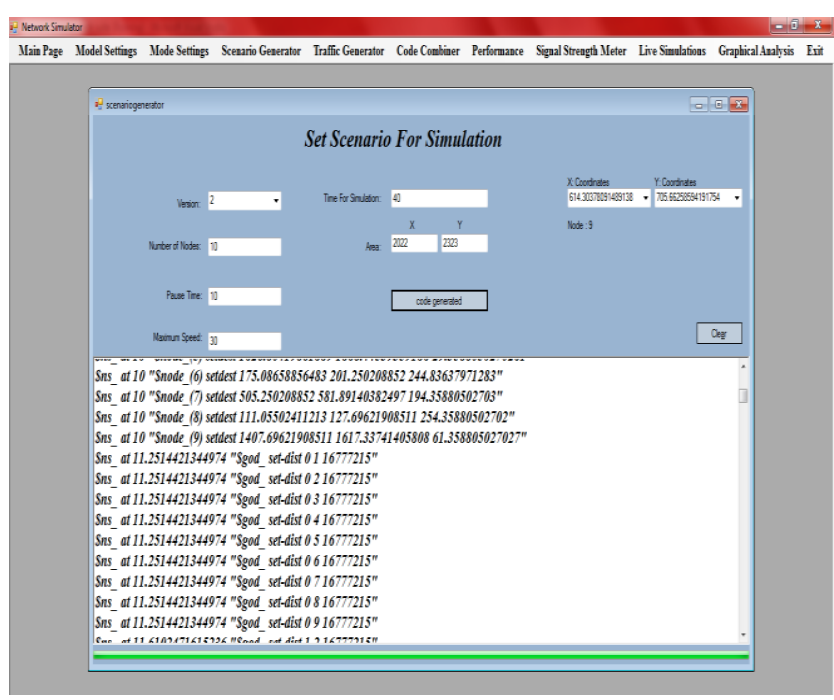

Fig. 7

Fig7. Indicates the scenario setter for performing simulations. This part of software can be used to generate the live simulations for that require multi line coding. The code for whole simulation is generated within few minutes. 


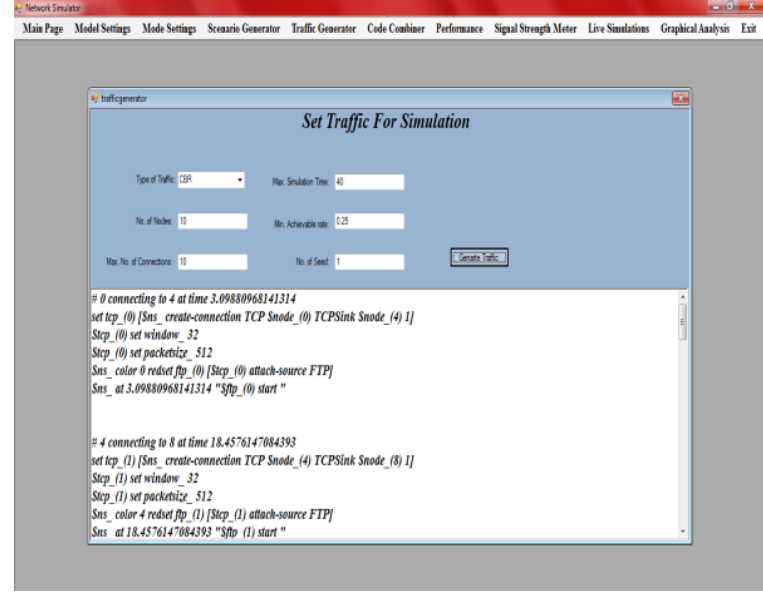

Fig. 8

Fig. 8 displays the traffic generator for simulations. This generates the code for connectivity between the nodes. The link can also be generated manually or automatically.

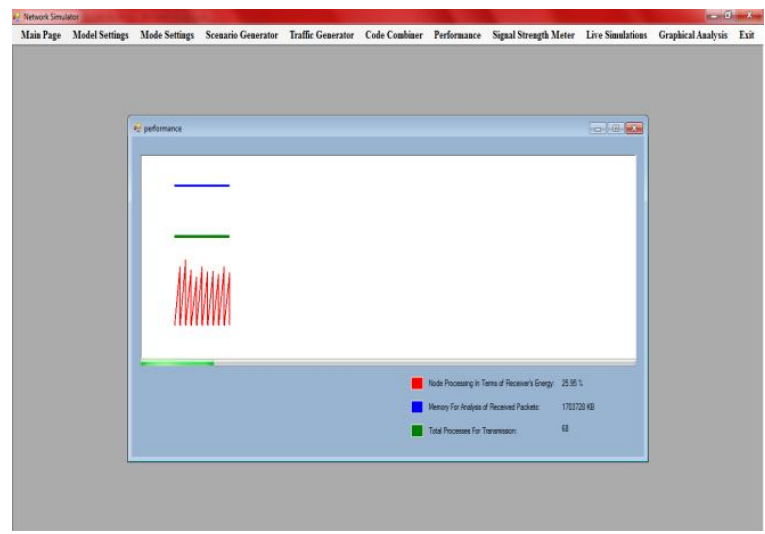

Fig. 9

This module generates the link budgeting evaluation by generating the live nodal graph in wave format along with the backend simulations that are being performed side by side.

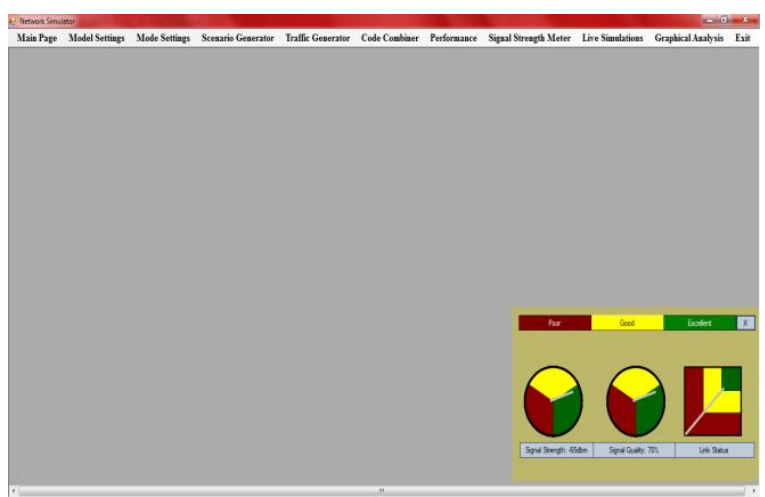

Fig. 10

Fig. 10 indicates the strength meter that shows the signal strength while simulations are being performed. It also shows the signal strength between the relaying nodes. This can be used to find out the weak and strong links between the nodes.

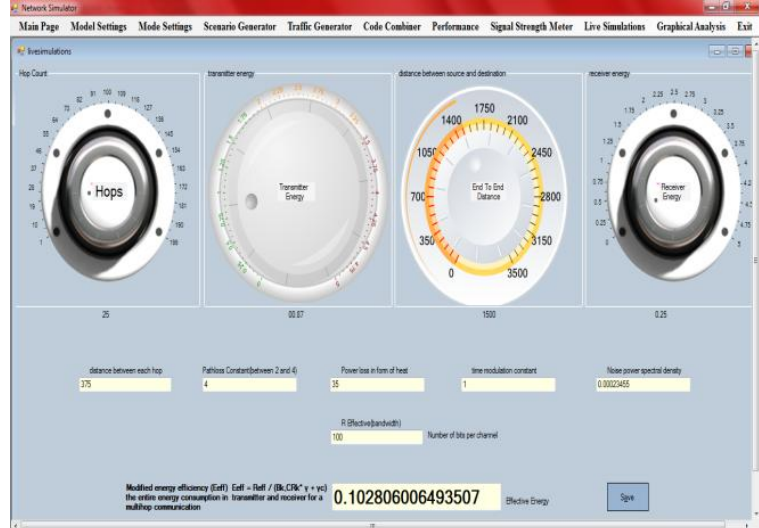

Fig. 11

Fig. 11 shows the live simulator module having knobs that can be used to generate live simulations even while previous simulations are running. This allows user to save result and implement it to already running simulations.

\subsection{Simulation Results and Analysis}

The graphical analysis is carried out by comparing the trace file of the newly designed and previous version of protocol. The comparison is carried out by use of files present in the xgraph of NS-2. The graphs taken by us are as follows:

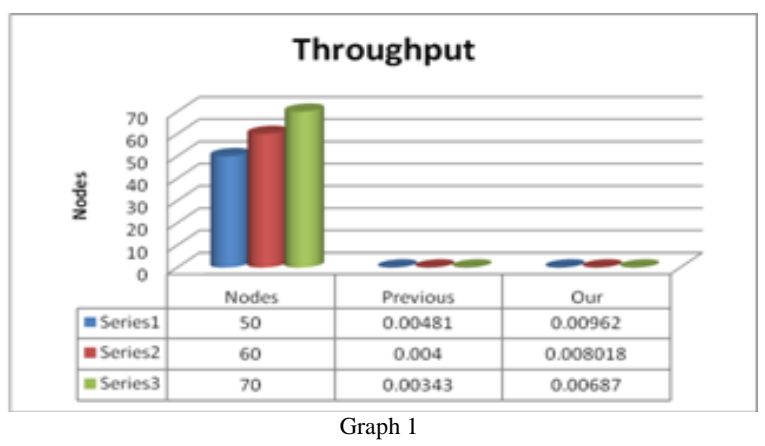

The graph 1 explains the throughput comparison between the DEPAS and the AODV protocol.

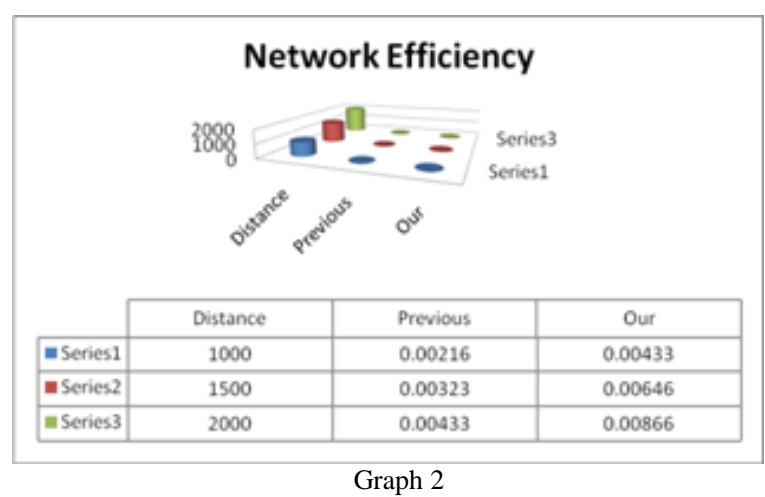

The graph 2 explains the graphical compariosn betweent he network effieicny of the AODV and the DEPAS protocol. 


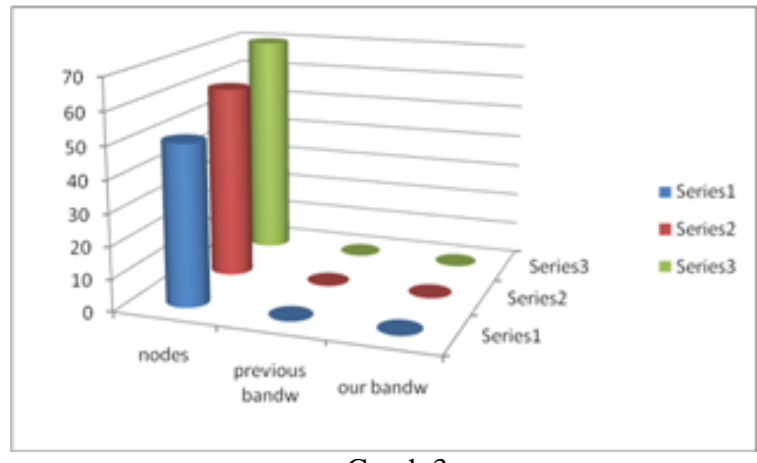

Graph 3

The Graph 3 explains the comparison between the AODV and DEPAS's Network Bandwidth.

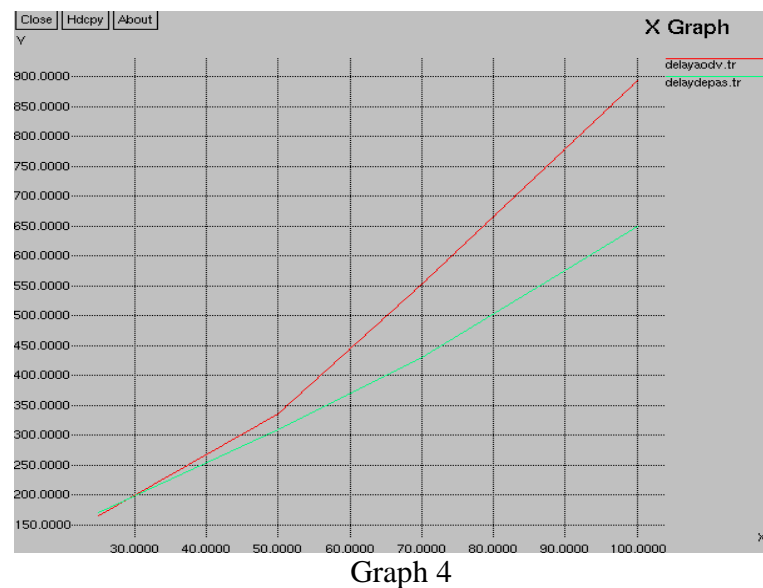

Graph 4 shows the relation between the DEPAS and the AODV protocol. Clearly, the DEPAS stands out to be better in the comparison of the two.

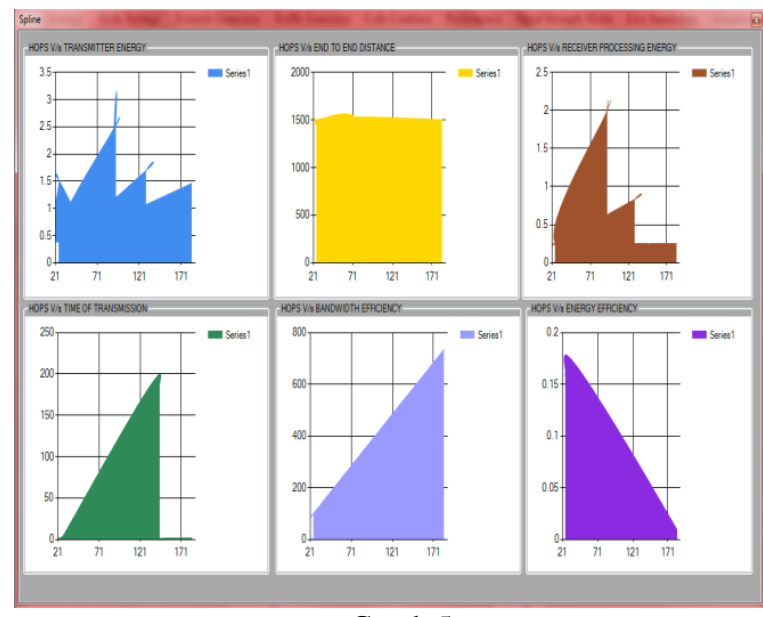

Graph 5

Graph 5 is the graphical analysis carried out by the simulator developed by us. It also shows the comparison of the results taken from NS-2 and itself. The graph generated by it are totally authenticated and justified.

Table 1.Our Simulator results

\begin{tabular}{|l|l|}
\hline Parameters & Improvement \\
\hline Transmitter Energy & $40 \%$ \\
\hline Reveiver Procesing Energy & $40 \%$ \\
\hline Energy Efficiency & $50 \%$ \\
\hline
\end{tabular}

\begin{tabular}{|l|l|}
\hline Bandwidth Efficiency & $50 \%$ \\
\hline Delays & Reduced By 65\% \\
\hline Working Efficiency & $85 \%$ \\
\hline
\end{tabular}

The above table shows the result of our simulator when compared with those taken using NS-2 simulator.

\section{CONCLUSION}

Thus, from the paper, it is noticed that in order to increase the performance of the network, the delays of the system must be decreased. In this paper, we have proposed DEPAS protocol. The simulator developed by us can be used to improve the quality of ad hoc networks. As shown in the analysis carried out by us using both ns- 2 and our own developed simulator, we claim that the proposed model is capable of eliminating the delays of the network structure consisting of mobile nodes. Also the power consumption of the network has been minimized with increased efficiency there by resulting in increased lifetime of the network structure. Further, work can be carried out to reduce the number of retransmissions that will further optimize the network structure.

\section{REFERENCES}

[1] Dr. R.K. Singh, Tanu Preet Singh, Vishal Sharma:"Dead State Recovery Based Power Optimization Routing Protocol for MANETs", HPAGC-2011, CCIS 169, pp.424-429,2011. (C) Springer-Verlag Berlin Heidelberg2011.

[2] Sunil Taneja and Ashwani Kush:" A Survey of Routing Protocols in Mobile Ad Hoc abstract". International Journal of Innovation, Management and Technology, Vol. 1, No. 3, August 2010.

[3] M. Saravana karthikeyan, M. Murali, Dr. S. Sujatha: "Identifying performance metrics to maximize Manet's throughput"; 2010 International Conference on Advances in Computer Engineering.

[4] Manika Vinay Rali, Min Song, Sachin Shetty:"Virtual wired transmission scheme using directional antennas to improve energy efficiency in Wireless Mobile Ad Hoc Network"; 978-1-4244-2677-5, IEEE 2008.

[5] Computational Science @ the University of Oregon : http://www. csi.uoregon.edu.

[6] Sehoon Kim, Jinkyu Lee and Ikjun Yeom," Modeling and Performance Analysis of Address Allocation Schemes for Wireless sensor networks", IEEE transactions on vehicular technology, vol. 57 , NO. 1 , JANUARY 2008.

[7] Rekha Patil, Dr. A. Damodaram:"cost basedd power aware cross layer routing protocol for Manet"; 2008 IJCSNS.

[8] Changchun Bae and Wayne E. Stark:"A Tradeoff between Energy and Bandwidth Efficiency in Wireless Networks"; 2007 IEEE.

[9] V. Rodoplu and T. H. Meng: "Bits-per-Joule capacity of energy-limited wireless networks," IEEE Transaction Wireless Communications, vol.6(3), pp.857-865, March 2007.

[10] B. Rankov and A. Wittneben: "Spectral efficient protocols for half-duplex fading relay channels," IEEE Journal on Selected Areas in Communications, vol. 25, pp.379-389: Feb. 2007.

[11] Network simulator-2 www.isi.edu/nanam/ns/. 